\title{
Eigenfunctions for singular fully nonlinear equations in unbounded domains
}

\author{
I. Birindelli and F. Demengel
}

\begin{abstract}
In this paper, we prove the existence of a generalized eigenvalue and a corresponding eigenfunction for fully nonlinear elliptic operators singular or degenerate, homogeneous of degree $1+\alpha, \alpha>-1$ in unbounded domains of $\mathbb{R}^{N}$. The main tool will be Harnack's inequality.
\end{abstract}

Mathematics Subject Classification (2000). 35J60, 35J75, 35P30.

\section{Introduction}

In this paper we prove the existence of a generalized eigenvalue and a corresponding eigenfunction for fully nonlinear operators singular or degenerate, homogeneous of degree $1+\alpha, \alpha>-1$ in unbounded domains of $\mathbb{R}^{N}$. The main tool will be the Harnack's inequality. The key hypotheses on the operator, homogeneity (H1) and ellipticity (H2) are given later.

Very recently Davila et al. [14,15] proved Harnack's inequality in all dimensions $N$ but in the singular case i.e. $\alpha<0$. Here, in the two dimensional case, we prove Harnack's inequality for any $\alpha>-1$. The proof uses in an essential way this dimensional restriction. It follows the lines of the original proof of Serrin [25] in the linear case. For Harnack's inequalities in quasilinear cases see [26] and [27]. Very recently Imbert [17] has proved an Harnack's inequality for fully nonlinear degenerate elliptic operators; let us mention that the class of operators he considers does not include those treated in this paper (see also [16] for degenerate elliptic equations in divergence form).

It is well known that Harnack's inequality gives a control on the oscillations of the solutions and hence it is used to prove uniform Hölder's estimates. It has been generalized to many 'weak' and nonlinear context, we are thinking for example about those due to Krylov and Safonov for "strong solutions" [20,21], or the results of Caffarelli, Cabré [12] for fully nonlinear equations that are uniformly elliptic.

Let us mention that in previous works on singular or degenerate fully nonlinear operators $[4,5]$ we proved Hölder's regularity of the solutions of 
Dirichlet problems in bounded domains. There the proof relied on the regularity of the solution on the boundary and the supremum of the solution. Hence in unbounded domains that tool cannot be used.

In the case treated here of fully nonlinear operators homogenous of degree $1+\alpha$, the Harnack inequality, due to Davila, Felmer and Quaas [14], is the following

Suppose that $F$ does not depend on $x$ and it satisfies

(H1) and (H2) as defined later and that $-1<\alpha \leq 0$. Suppose that $V, h$ and $f$ are continuous and that $u$ is a nonnegative solution of

$$
F\left(\nabla u, D^{2} u\right)+h(x) \cdot \nabla u|\nabla u|^{\alpha}+V(x) u^{1+\alpha}=f \quad \text { in } \Omega .
$$

Then for all $\Omega^{\prime} \subset \subset \Omega$ there exists some constant $C$ which depends on $a, A, \alpha$, $V, h, N, \Omega^{\prime}, \Omega$, such that

$$
\sup _{\Omega^{\prime}} u \leq C\left(\inf _{\Omega^{\prime}} u+\|f\|_{L^{N}\left(\Omega^{\prime}\right)}^{\frac{1}{1+\alpha}}\right) .
$$

Among all the consequences of Harnack's inequality, Berestycki et al. in their acclaimed paper [1] proved the existence of an eigenfunction for a linear, uniformly elliptic operator when no regularity of the boundary of the domain is known. The idea being that, close to the boundary, the solutions are controlled by the maximum principle in "small" domains, and, in the interior, one can use Harnack's inequality.

As it is well known, inspired by [1], the concept of eigenvalue in the case of bounded regular domains has lately been extended to fully nonlinear operators (see $[4,5,7,18,22-24]$ ). Two "principal eigenvalues" can be defined as the extremum of the values for which the maximum principle or respectively the minimum principle holds.

In this article we want to use the Harnack inequality obtained here and in $[14,15]$ (see also [8]) to study the eigenvalue problem in unbounded domains. Let us recall that in general, even for the Laplacian operator, the maximum principle does not hold in unbounded domains, hence we cannot define the "principal" eigenvalue in the same way as in the case of bounded domains. In [10] and [11] Capuzzo Dolcetta, Leoni and Vitolo study the conditions on the domain $\Omega$ in order for the Maximum principle to hold for fully nonlinear operators, extending the result of Cabré [9].

Furthermore let us mention that in unbounded domains, even for the Laplacian, there are several possible definitions of "eigenvalues" as the reader can see in Berestycki and Rossi [2]. Here we define the first eigenvalue as the infimum of the first eigenvalues for bounded smooth domains included in $\Omega$. We prove the existence of a positive eigenfunction for this so called eigenvalue, using Harnack's inequality.

We shall also prove the existence of solutions for equations below the eigenvalues. Observe that differently from the case of bounded domains, we can't use the maximum principle since in general it won't hold.

The paper is organized as follows. In the next section we state the main assumptions and some key theorems, in Sect. 3 we state precisely the main 
results i.e. Harnack's inequality and existence of solutions in unbounded domains. Finally the proofs are given in the last section.

\section{Assumptions and known results}

\subsection{Hypotheses}

Let $\Omega$ be a domain of $\mathbb{R}^{N}$. In the whole paper we consider solutions of the equation

$$
F\left(x, \nabla u, D^{2} u\right)+h(x) \cdot \nabla u|\nabla u|^{\alpha}+V(x) u^{1+\alpha}=f(x) \quad \text { in } \Omega,
$$

with the following hypotheses on $F, h$ and $V$ :

Let $\alpha>-1$ and $S$ be the set of symmetric $N \times N$ matrices:

(H1) $F$ is continuous on $\Omega \times \mathbb{R}^{N} \backslash\{0\} \times S \rightarrow \mathbb{R}$, and $\forall t \in \mathbb{R} \backslash\{0\}, \mu \geq 0$,

$$
F(x, t p, \mu X)=|t|^{\alpha} \mu F(x, p, X) .
$$

(H2) There exists $0<a \leq A$ such that for all $p \in \mathbb{R}^{N} \backslash\{0\}, M \in S, N \in S$, $N \geq 0$

$$
a|p|^{\alpha} \operatorname{tr}(N) \leq F(x, p, M+N)-F(x, p, M) \leq A|p|^{\alpha} \operatorname{tr}(N) .
$$

(H3) There exists a continuous function $\omega$ with $\omega(0)=0$, such that if $(X, Y) \in S^{2}$ and $\zeta \in \mathbb{R}^{+}$satisfy

$$
-\zeta\left(\begin{array}{ll}
I & 0 \\
0 & I
\end{array}\right) \leq\left(\begin{array}{cc}
X & 0 \\
0 & Y
\end{array}\right) \leq 4 \zeta\left(\begin{array}{cc}
I & -I \\
-I & I
\end{array}\right)
$$

and $I$ is the identity matrix in $\mathbb{R}^{N}$, then for all $(x, y) \in \mathbb{R}^{N}, x \neq y$,

$$
F(x, \zeta(x-y), X)-F(y, \zeta(x-y),-Y) \leq \omega\left(\zeta|x-y|^{2}\right) .
$$

Observe that when $F$ is independent of $x$, condition (H3) is a consequence of $(\mathrm{H} 2)$.

We assume that $h$ and $V$ are some continuous bounded functions on $\bar{\Omega}$ and $h$ satisfies

(H4) - Either $\alpha \leq 0$ and $h$ is Hölder continuous of exponent $1+\alpha$,

- or $\alpha>0$ and

$$
(h(x)-h(y)) \cdot(x-y) \leq 0 .
$$

Recall that examples of operators satisfying these conditions include the $p$ Laplacian with $\alpha=p-2$ and

$$
F\left(\nabla u, D^{2} u\right)=|\nabla u|^{\alpha} \mathcal{M}_{a, A}^{ \pm}\left(D^{2} u\right)
$$

where $\mathcal{M}_{a, A}^{+}$is the Pucci operator $\mathcal{M}_{a, A}^{+}(M)=A \operatorname{Tr}\left(M^{+}\right)-a \operatorname{Tr}\left(M^{-}\right)$and $\mathcal{M}_{a, A}^{-}(M)=a \operatorname{Tr}\left(M^{+}\right)-A \operatorname{Tr}\left(M^{-}\right)$.

For another example, let $\alpha \leq 0, B$ be some matrix with Lipschitz coefficients, and invertible for all $x \in \Omega$. Let us consider $A(x)=B^{\star} B(x)$ and the operator 


$$
F(x, p, M)=|p|^{\alpha}(\operatorname{tr}(A(x)(M)) .
$$

Then $F$ satisfies $(\mathrm{H} 1), \ldots,(\mathrm{H} 3)$.

Remark 2.1. When no ambiguity arises we shall sometimes write $F[u]$ to signify $F\left(x, \nabla u, D^{2} u\right)$.

The solutions that we consider will be taken in a generalized viscosity sense see e.g. [3] for precise definitions, let us recall that in particular we do not test when the gradient of the test function is null.

\subsection{Known results in bounded domains}

We assume in this subsection that $\Omega$ is a bounded domain.

We first recall a weak comparison principle (see [3]), which will be used in the proof of Theorem 3.1.

Theorem 2.2. Suppose that $f$ and $g$ are continuous and bounded and that $u$ and $v$ satisfy

$$
\begin{gathered}
F\left(x, \nabla u, D^{2} u\right)+h(x) \cdot \nabla u|\nabla u|^{\alpha}+V(x)|u|^{\alpha} u \geq g \quad \text { in } \Omega, \\
F\left(x, \nabla v, D^{2} v\right)+h(x) \cdot \nabla v|\nabla v|^{\alpha}+V(x)|v|^{\alpha} v \leq f \quad \text { in } \Omega, \\
u \leq v \quad \text { on } \partial \Omega .
\end{gathered}
$$

If $V \leq 0$ and $f<g$, then $u \leq v$ in $\Omega$.

If $V<0$ and $f \leq g$, then $u \leq v$ in $\Omega$.

We shall also need for the proof of Theorem 3.1 another comparison principle:

Theorem 2.3. [5] Suppose that $\tau<\bar{\lambda}(\Omega), f \leq 0, f$ is upper semi-continuous and $g$ is lower semi-continuous with $f \leq g$.

Let $u$ and $v$ be respectively continuous solutions of

$$
\begin{aligned}
& F\left(x, \nabla u, D^{2} u\right)+h(x) \cdot \nabla u|\nabla u|^{\alpha}+(V(x)+\tau)|u|^{\alpha} u \geq g \text { in } \Omega, \\
& F\left(x, \nabla v, D^{2} v\right)+h(x) \cdot \nabla v|\nabla v|^{\alpha}+(V(x)+\tau) v^{1+\alpha} \leq f \quad \text { in } \Omega, \\
& u \leq v \quad \text { on } \partial \Omega \text {, }
\end{aligned}
$$

with $v \geq 0$.

In each of these three cases:

1) $v>0$ on $\bar{\Omega}$ and $f<0$ in $\Omega$,

2) $v>0$ on $\bar{\Omega}$ and $g(x)>0$ wherever $f(x)=0$,

3) $v>0$ in $\Omega, f<0$ in $\bar{\Omega}$ and $f<g$ on $\bar{\Omega}$,

we have that $u \leq v$ in $\Omega$.

We also recall the following regularity result:

Proposition 2.4. [5] Let $\Omega$ be a smooth domain. Let $f$ be a continuous function in $\bar{\Omega}$. Let $u$ be a viscosity non-negative bounded solution of

$$
\left\{\begin{array}{lc}
F\left(x, \nabla u, D^{2} u\right)+h(x) \cdot \nabla u|\nabla u|^{\alpha}=f & \text { in } \Omega, \\
u=0 & \text { in } \partial \Omega .
\end{array}\right.
$$


Then, for any $\gamma<1$, there exists a constant $C$ which depends only on $|f|_{\infty}$, $|h|_{\infty}$ and $|u|_{\infty}$ such that :

$$
|u(x)-u(y)| \leq C|x-y|^{\gamma}
$$

for any $(x, y) \in \bar{\Omega}^{2}$.

\section{Main results}

\subsection{Harnack's inequality in the two dimensional case}

In this subsection we state Harnack's inequality, together with some important corollary. These results will be proved in Sect. 4 and used in the next subsection.

Theorem 3.1 (Harnack's inequality). Suppose that $\Omega$ is a bounded domain in $\mathbb{R}^{2}$ and that $f$ is continuous on $\bar{\Omega}$. Let $u$ be a positive solution of

$$
F\left(x, \nabla u, D^{2} u\right)+h(x) \cdot \nabla u|\nabla u|^{\alpha}+V(x) u^{1+\alpha}=f(x) \quad \text { in } \Omega .
$$

Let $\Omega^{\prime} \subset \subset \Omega$. Then there exists $K=K\left(\Omega, \Omega^{\prime}, A, a,|h|_{\infty},|V|_{\infty}\right)$ such that

$$
\sup _{\Omega^{\prime}} u \leq K\left(\inf _{\Omega^{\prime}} u+|f|_{\infty}^{\frac{1}{1+\alpha}}\right) .
$$

Corollary 3.2. Let $u$ be a positive solution of (3.3). Let $R_{o}$ be such that $B(0$, $\left.R_{o}\right) \subset \Omega$. Then there exists $K$ which depends only on $A, a,|h|_{\infty}$ and $R_{o}$, such that for any $R<R_{o}$ :

$$
\sup _{B(0, R)} u \leq K\left(\inf _{B(0, R)} u+R^{\frac{2+\alpha}{1+\alpha}}|f|_{\infty}^{\frac{1}{1+\alpha}}\right) .
$$

As a consequence, for any solution $u$ of (3.3) and for any $\Omega^{\prime} \subset \subset \Omega$, there exists $\beta \in(0,1)$ depending on Harnack's constant $K$ in $(3.5)$ such that $u \in C^{o, \beta}\left(\Omega^{\prime}\right)$.

An immediate consequence of Harnack's inequality is the following Liouville type result:

Corollary 3.3 (Liouville). Let $u$ be a solution of $F\left(x, \nabla u, D^{2} u\right)=0$ in $\mathbb{R}^{2}$, if $u$ is bounded from below, then $u$ is constant.

See [13] for other Liouville results.

\subsection{Existence's results in unbounded domains}

Before stating the results in unbounded domains, we recall what we mean by first eigenvalue and the property of these eigenvalues in the bounded case.

When $\Omega$ is a bounded domain we define

$$
\begin{aligned}
\bar{\lambda}(\Omega)=\sup \{\lambda, \exists \varphi \in \mathcal{C}(\bar{\Omega}), \varphi>0 \text { in } \Omega, \\
\left.F[\varphi]+h(x) \cdot \nabla \varphi|\nabla \varphi|^{\alpha}+(V(x)+\lambda) \varphi^{1+\alpha} \leq 0\right\},
\end{aligned}
$$

and

$$
\begin{aligned}
\underline{\lambda}(\Omega)=\sup \{\lambda, \exists \varphi \in \mathcal{C}(\bar{\Omega}), \varphi<0 \quad \text { in } \Omega, \\
\left.F[\varphi]+h(x) \cdot \nabla \varphi|\nabla \varphi|^{\alpha}+(V(x)+\lambda) \varphi|\varphi|^{\alpha} \geq 0\right\} .
\end{aligned}
$$


We proved in [5] that when $\Omega$ is a smooth bounded domain, there exists $\varphi>0$ and $\psi<0$ in $\Omega$ which are respectively a solution of

$$
\begin{cases}F[\varphi]+h(x) \cdot \nabla \varphi|\nabla \varphi|^{\alpha}+(V(x)+\bar{\lambda}(\Omega)) \varphi^{1+\alpha}=0 & \text { in } \Omega, \\ \varphi=0 & \text { on } \partial \Omega\end{cases}
$$

and

$$
\begin{cases}F[\psi]+h(x) \cdot \nabla \psi|\nabla \psi|^{\alpha}+(V(x)+\underline{\lambda}(\Omega))|\psi|^{\alpha} \psi=0 & \text { in } \Omega, \\ \psi=0 & \text { on } \partial \Omega .\end{cases}
$$

Moreover $\varphi$ and $\psi$ are Hölder continuous as recalled in Proposition 2.4.

When $\Omega \subset \mathbb{R}^{N}$ is unbounded, we extend in the following way the definition of the "eigenvalues":

$$
\bar{\lambda}(\Omega)=\inf \{\bar{\lambda}(A), \text { for all smooth bounded domain } A, A \subset \Omega\},
$$

and

$$
\underline{\lambda}(\Omega)=\inf \{\underline{\lambda}(A) \text {, for all smooth bounded domain } A, A \subset \Omega\} .
$$

When no ambiguity arises we shall omit to write the dependence of the eigenvalues with respect to the domain $\Omega$.

We start by giving some lower bounds on the eigenvalues. For simplicity this will be done for $h \equiv 0, V \equiv 0$. If $\Omega$ is bounded it is easy to see that $\bar{\lambda}(\Omega)>0$, while it is obvious that for $\Omega=\mathbb{R}^{N}, \bar{\lambda}(\Omega)=0$. We wish to prove that this is not the case for all unbounded domains, in fact we shall see that if $\Omega$ is bounded in one direction, then $\bar{\lambda}(\Omega)>0$.

Proposition 3.4. Suppose that $\Omega$ is contained in a strip of width $M$ i.e. up to translation and rotation

$$
\Omega \subset[0, M] \times \mathbb{R}^{N-1}
$$

then there exists $C=C(\alpha, a)>0$ such that

$$
\bar{\lambda}(\Omega) \geq \frac{C}{M^{2+\alpha}} .
$$

Proof. Let $u(x)=4 M^{2}-\left(x_{1}+M\right)^{2}$. Then $u^{1+\alpha} \leq\left(3 M^{2}\right)^{1+\alpha}$ and $F[u] \leq$ $-2^{1-|\alpha|} M^{\alpha} a$ in $[0, M] \times \mathbb{R}^{N-1}$. Hence $\bar{\lambda}(\Omega) \geq \frac{2^{1-|\alpha|}}{3^{1+\alpha} M^{2+\alpha} a}$. This gives (3.6) and it ends the proof.

In the next theorem, we want to be in the same hypotheses for which Harnack's inequality is known, hence we consider the following condition:

(C) Either $N \geq 3, F$ is independent of $x$ and $-1<\alpha \leq 0$; or $N=2, \alpha>-1$ and $F$ may depend on $x$.

Theorem 3.5. Suppose that $\Omega$ is a (possibly unbounded) domain of $\mathbb{R}^{N}$. Suppose that $F$ satisfies $(C)$. Then there exists a positive function $\phi$, respectively a negative function $\psi$, which is a solution of

$$
F[\phi]+h(x) \cdot \nabla \phi|\nabla \phi|^{\alpha}+(V(x)+\bar{\lambda}(\Omega)) \phi^{1+\alpha}=0 \quad \text { in } \Omega,
$$

respectively

$$
F[\psi]+h(x) \cdot \nabla \psi|\nabla \psi|^{\alpha}+(V(x)+\underline{\lambda}(\Omega))|\psi|^{\alpha} \psi=0 \quad \text { in } \Omega .
$$

Furthermore $\phi$ and $\psi$ are Hölder continuous. 
Remark 3.6. In Theorem 3.5, we do not require that $\phi$ and $\psi$ be zero on $\partial \Omega$. Nonetheless $\inf _{\Omega} \phi=\inf _{\Omega}|\psi|=0$ otherwise it would contradict the definition of eigenvalues.

In the next proposition we prove some existence's result "below" the eigenvalues.

Proposition 3.7. For any $\lambda<\bar{\lambda}(\Omega)$ (respectively $\lambda<\underline{\lambda}(\Omega)$ ), for any $f \in \mathcal{C}_{c}(\Omega)$ non positive (respectively non negative), there exists a solution $v>0$ (respectively $v<0$ ) of

$$
F[v]+h(x) \cdot \nabla v|\nabla v|^{\alpha}+(V(x)+\lambda)|v|^{\alpha} v=f \quad \text { in } \Omega .
$$

Furthermore, in both cases, for $f \not \equiv 0$ there exists $C$ such that

$$
|v|_{\infty} \leq C|f|_{\infty}^{\frac{1}{1+\alpha}}
$$

Remark 3.8. As mentioned in Proposition 2.4, we proved some Hölder's regularity result for all $\beta \in[0,1$ [ in bounded regular domains for homogeneous or regular boundary conditions, [4]. More precisely the Hölder's constants depend on the $L^{\infty}$ norm of $u$ and $u$ is zero on the boundary. This gives also some Hölder's uniform estimates for sequences of solutions. As a consequence, a sequence of solutions converges, for a subsequence, towards a solution. This cannot be used in the proof of the results above, indeed we shall need compactness results inside bounded sets $\Omega_{n}$ whose size increases, for sequence of functions which have uniform $L^{\infty}$ bounds on bounded fixed sets, but for which the $L^{\infty}\left(\Omega_{n}\right)$ norm may go to infinity.

\section{Proofs of the main results}

\subsection{Proof of existence}

We start by the existence of some "eigenfunction".

Proof of Theorem 3.5. We shall only give the proof for the positive eigenfunction $\phi$, the case of the negative eigenfunction $\psi$ being analogous. Let $\left(\Omega_{n}\right)_{n}$ be a sequence of smooth, bounded domains such that

$$
\Omega_{n} \subset \subset \Omega_{n+1} \subset \subset \Omega, \quad \bar{\lambda}\left(\Omega_{n}\right) \rightarrow \bar{\lambda}(\Omega) \text { and } \cup_{n} \Omega_{n}=\Omega .
$$

Let $f_{n}$ be a sequence of functions in $\mathcal{C}_{c}\left(\Omega_{n} \backslash \overline{\Omega_{n-1}}\right), f_{n} \leq 0$ and not identically zero. Since $\bar{\lambda}\left(\Omega_{n}\right)>\bar{\lambda}(\Omega)$, for any $n$, the existence's results in [5] ensure that there exists $u_{n}>0$ in $\Omega_{n}$ which solves

$$
\begin{cases}F\left[u_{n}\right]+h(x) \cdot \nabla u_{n}\left|\nabla u_{n}\right|^{\alpha}+(\bar{\lambda}(\Omega)+V(x)) u_{n}^{1+\alpha}=f_{n} & \text { in } \Omega_{n}, \\ u_{n}=0 & \text { on } \partial \Omega_{n} .\end{cases}
$$

Fix $x_{0} \in \Omega_{1}$, let

$$
v_{n}(x)=\frac{u_{n}(x)}{u_{n}\left(x_{0}\right)}, \quad \text { for all } x \in \Omega_{n} .
$$

We extend them to zero in $\Omega \backslash \overline{\Omega_{n}}$, obtaining in such a way a sequence of continuous functions in $\Omega$. Let $O^{\prime}, O$ be regular domains such that $O \subset \subset O^{\prime} \subset \subset \Omega$. 
We prove that $\left(v_{n}\right)_{n}$ converges uniformly on $K^{\prime}=\bar{O}^{\prime}$. Indeed there exists $N_{0}$ such that for all $n \geq N_{0}, \Omega_{n}$ contains $K^{\prime}$. As a consequence we have, for such $n$,

$$
F\left[v_{n}\right]+h(x) \cdot \nabla v_{n}\left|\nabla v_{n}\right|^{\alpha}+(V(x)+\bar{\lambda}(\Omega)) v_{n}^{1+\alpha}=0 \quad \text { in } O^{\prime}
$$

Moreover $v_{n}\left(x_{0}\right)=1$. By Harnack's inequality of Theorem 3.1 there exists a constant $C_{K^{\prime}}$ such that

$$
\sup _{K^{\prime}} v_{n} \leq C_{K^{\prime}}\left(\inf _{K^{\prime}} v_{n}\right) \leq C_{K^{\prime}} .
$$

This implies in particular that $v_{n}$ is bounded independently of $n$ in $K^{\prime}$.

Using Corollary 3.2 on the open set $O^{\prime}$, one gets that $\left(v_{n}\right)_{n}$ is relatively compact in $\mathcal{C}(\bar{O})$. A subsequence of $\left(v_{n}\right)_{n}$ will converge towards $\phi$, a solution of

$$
F[\phi]+h(x) \cdot \nabla \phi|\nabla \phi|^{\alpha}+(V(x)+\bar{\lambda}(\Omega)) \phi^{1+\alpha}=0 \quad \text { in } O .
$$

Since $\phi\left(x_{0}\right)=\lim v_{n}\left(x_{0}\right)=1, \phi$ cannot be identically zero. By the strong maximum principle on compact subdomains of $\Omega, \phi>0$ inside $\Omega$. Since $O$ is an arbitrary subdomain of $\Omega$, this ends the proof.

Proof of Proposition 3.7. We consider only the case $f \leq 0$ and $\lambda<\bar{\lambda}(\Omega)$. We first treat $f \not \equiv 0$. Let $K$ be the compact support of $f$. As in the previous proof let $\left(\Omega_{n}\right)_{n}$ be a sequence of bounded smooth domains, such that

$$
\Omega_{n} \subset \Omega_{n+1} \text { and } \cup_{n} \Omega_{n}=\Omega .
$$

Since $\bar{\lambda}\left(\Omega_{n}\right) \geq \bar{\lambda}(\Omega)$, according to the existence's results in [5], there exists $u_{n}$, a positive solution of

$$
\begin{cases}F\left[u_{n}\right]+h(x) \cdot \nabla u_{n}\left|\nabla u_{n}\right|^{\alpha}+(V(x)+\lambda) u_{n}^{1+\alpha}=f & \text { in } \Omega_{n}, \\ u_{n}=0 & \text { on } \partial \Omega_{n} .\end{cases}
$$

Let $\varphi^{+}$be the function given in Theorem 3.5 such that

$$
F\left[\varphi^{+}\right]+h(x) \cdot \nabla \varphi^{+}\left|\nabla \varphi^{+}\right|^{\alpha}+(V(x)+\bar{\lambda}(\Omega))\left(\varphi^{+}\right)^{1+\alpha}=0 \quad \text { in } \Omega,
$$

with $\max _{K} \varphi^{+}=1$. By homogeneity, the function

$$
\varphi_{1}(x)=\frac{\sup |f|^{\frac{1}{1+\alpha}}}{(\bar{\lambda}-\lambda)^{\frac{1}{1+\alpha}} \inf _{K} \varphi^{+}} \varphi^{+}(x),
$$

is a solution of

$$
F\left[\varphi_{1}\right]+h(x) \cdot \nabla \varphi_{1}\left|\nabla \varphi_{1}\right|^{\alpha}+(V(x)+\lambda) \varphi_{1}^{1+\alpha}=(\lambda-\bar{\lambda}) \frac{\left(\varphi^{+}\right)^{1+\alpha} \sup (-f)}{(\bar{\lambda}-\lambda)\left(\inf _{K} \varphi^{+}\right)^{1+\alpha}} \leq f .
$$

Since $\varphi_{1}>0$ on $\partial \Omega_{n}$, the comparison principle in Theorem 2.3, gives for any $n$

$$
0 \leq u_{n} \leq \varphi_{1} \quad \text { in } \Omega_{n}
$$

The same argument as in the proof of Theorem 3.5 shows that there is a subsequence of $\left(u_{n}\right)_{n}$, converging uniformly on every compact subset of $\Omega$, to a solution $u$ of

$$
F[u]+h(x) \cdot \nabla u|\nabla u|^{\alpha}+(V(x)+\lambda) u^{1+\alpha}=f \quad \text { in } \Omega .
$$


The strong maximum principle applied on bounded subdomains of $\Omega$ implies that $u>0$.

We now consider the case $f \equiv 0$. We only give the proof in the case $\lambda<\bar{\lambda}(\Omega)$, the other case being analogous.

Let $\left(\Omega_{n}\right)_{n}$ be a sequence of smooth bounded domains such that

$$
\Omega_{n} \subset \Omega_{n+1} \text { and } \cup_{n} \Omega_{n}=\Omega \text {. }
$$

For any $n$, let $u_{n}$ be the solution of

$$
\begin{cases}F\left[u_{n}\right]+h(x) \cdot \nabla u_{n}\left|\nabla u_{n}\right|^{\alpha}+(V(x)+\lambda) u_{n}\left|u_{n}\right|^{\alpha}=0 & \text { in } \Omega_{n}, \\ u_{n}=1 & \text { on } \partial \Omega_{n} .\end{cases}
$$

Since $\lambda<\bar{\lambda}\left(\Omega_{n}\right), u_{n}$ is well defined and $u_{n}>0$ in $\Omega_{n}$ (see [6]).

Fix $x_{0} \in \Omega_{1}$. Observe that $v_{n}=\frac{u_{n}}{u_{n}\left(x_{0}\right)}$ is a solution of

$$
F\left[v_{n}\right]+h(x) \cdot \nabla v_{n}\left|\nabla v_{n}\right|^{\alpha}+(V(x)+\lambda) v_{n}^{1+\alpha}=0 .
$$

By Harnack's inequality, for every $O$ such that $O \subset \subset \Omega,\left(v_{n}\right)_{n}$ is bounded on $K=\bar{O}$.

Using Corollary 3.2 as before, there exists a subsequence of $\left(v_{n}\right)_{n}$ which converges uniformly on every compact subdomain of $\Omega$, to $v$ which is a solution of

$$
F[v]+h(x) \cdot \nabla v|\nabla v|^{\alpha}+(V(x)+\lambda) v^{1+\alpha}=0 \quad \text { in } \Omega .
$$

Moreover $v\left(x_{0}\right)=1$, therefore $v$ is not identically zero. By the strong maximum principle $v>0$ in $\Omega$. This ends the proof.

\subsection{Proofs of Harnack's inequality in the two dimensional case}

The proof that we propose follows the lines of the proof of Serrin [25], see also Gilbarg Trudinger [19], with some new arguments that make explicit use of the eigenfunction in bounded domains. This extends the result of [14] to the case $\alpha>0$, but only in the two dimensional case.

In the proof of Theorem 3.1 we shall use the following

Lemma 4.1. Let $b$ and $c$ be some positive numbers, $x_{o}=\left(x_{o_{1}}, x_{o_{2}}\right) \in \mathbb{R}^{2}$. Let

$E=\left\{x=\left(x_{1}, x_{2}\right), \sigma^{2}(x):=\frac{\left(x_{1}-x_{o_{1}}\right)^{2}}{b^{2}}+\frac{\left(x_{2}-x_{o_{2}}\right)^{2}}{c^{2}} \leq 1, x_{1}-x_{o_{1}}>\frac{b}{2}\right\}$.

Then there exist two constants $\gamma>0$ and $\epsilon>0$ such that the function

$$
v(x)=\frac{e^{-\gamma \sigma^{2}(x)}-e^{-\gamma}}{e^{-\gamma / 4}-e^{-\gamma}}
$$


satisfies

$$
F[v]-|h|_{\infty}|\nabla v|^{1+\alpha}-|V|_{\infty} v^{1+\alpha}>\epsilon \quad \text { in } E
$$

and $v=0$ on $\partial E \cap\left\{x_{1}-x_{o_{1}}>\frac{b}{2}\right\}$.

Remark 4.2. The same result holds for the symmetric part of ellipsis : $E=$ $\left\{x=\left(x_{1}, x_{2}\right), \sigma^{2}(x) \leq 1, x_{1}-x_{o_{1}}<\frac{-b}{2}\right\}$.

Proof of Lemma 4.1. Without loss of generality one can assume that $x_{o}=0$.

Let $\tilde{v}=\frac{e^{-\gamma \sigma^{2}}}{e^{-\gamma / 4}-e^{-\gamma}}$ and let $B$ be the diagonal $2 \times 2$ matrix, with $B_{11}=\frac{1}{b^{2}}$ and $B_{22}=\frac{1}{c^{2}}$. Then $\nabla v=-2 \gamma \tilde{v} B x$ and

$$
D^{2} v=(2 \gamma)(2 \gamma B x \otimes B x-B) \tilde{v} .
$$

Since $B$ and $B x \otimes B x$ are both nonnegative,

$$
\begin{aligned}
& a\left(\operatorname{tr}\left(D^{2} v\right)^{+}\right)-A\left(\operatorname{tr}\left(D^{2} v\right)^{-}\right) \\
& \quad \geq\left(a \gamma^{2} 4\left(\frac{x_{1}^{2}}{b^{4}}+\frac{x_{2}^{2}}{c^{4}}\right)-2(A+a) \gamma\left(\frac{1}{b^{2}}+\frac{1}{c^{2}}\right)\right) \tilde{v} .
\end{aligned}
$$

We define

$$
m=\inf \left(b^{-\alpha}, 2^{\alpha}\left(\frac{1}{b^{2}}+\frac{1}{c^{2}}\right)^{\alpha / 2}\right) \quad \text { and } \quad M=2^{1+\alpha}\left(\frac{1}{b^{2}}+\frac{1}{c^{2}}\right)^{\frac{1+\alpha}{2}} .
$$

Choosing

$$
\gamma=\sup \left(\frac{4(A+a)}{a}\left(1+\frac{b^{2}}{c^{2}}\right), \frac{4|h|_{\infty} M b^{2}}{a m},\left(\frac{4|V|_{\infty} b^{2}}{a m}\right)^{\frac{1}{2+\alpha}}\right),
$$

and using (H1), there exists $\varepsilon>0$ such that :

$$
\begin{aligned}
& F\left(x, \nabla v, D^{2} v\right)+h(x) \cdot \nabla v|\nabla v|^{\alpha}+V(x) v^{1+\alpha} \\
& \quad \geq|\nabla v|^{\alpha}\left(a\left(\operatorname{tr}\left(D^{2} v\right)^{+}\right)-A\left(\operatorname{tr}\left(D^{2} v\right)^{-}\right)\right)-|h|_{\infty}|\nabla v|^{1+\alpha}-|V|_{\infty} v^{1+\alpha} \\
& \quad \geq \varepsilon>0 .
\end{aligned}
$$

This ends the proof of Lemma 4.1 .

Proof of Theorem 3.1. Let us remark that $u \geq 0$ implies that $\bar{\lambda}(\Omega) \geq 0$, according to the definition of $\bar{\lambda}(\Omega)$. Moreover without loss of generality we can suppose that $\bar{\lambda}(\Omega)>0$. Indeed, using again the definition of the eigenvalue, there exists $\Omega_{1} \subset \Omega$ such that $\Omega^{\prime} \subset \subset \Omega_{1}$ and $\bar{\lambda}\left(\Omega_{1}\right)>\lambda(\Omega) \geq 0$. Then we consider the proof in $\Omega_{1}$ instead of $\Omega$.

The proof proceeds in the following way, we first prove (3.4) in a ball of radius 1 . Thanks to the homogeneity of the equation the inequality is extended to balls of any bounded radius $R$. Finally, for general bounded domains $\Omega,(3.4)$ is proved using an argument that is standard in potential theory. 
So we begin with the following

Claim: Suppose that $\Omega=B(0,1)$. There exists $K$ which depends only on $a$, $A$, and bounds on $h$ and $V$ such that

$$
u(0) \leq K\left(\min _{B\left(0, \frac{1}{3}\right)} u+|f|_{\infty}^{\frac{1}{1+\alpha}}\right) .
$$

Proof of the Claim: Since $\bar{\lambda}(B(0,1))>0$, for $\delta>0$ sufficiently small, $\bar{\lambda}_{\delta}:=$ $\bar{\lambda}(B(0,1+\delta))>0$ as well.

We show first that there exists a constant $\epsilon>0$ such that if $\max _{B(0,1)}|f| \leq$ $\epsilon$, then for some constant $K \geq 1$

$$
u(0) \leq K\left(\min _{B\left(0, \frac{1}{3}\right)} u+1\right) .
$$

If $u(0) \leq 1$, the inequality holds for every $K$ greater than 1 . We assume then that $u(0)>1$.

Let $\phi_{\delta}$ be some positive eigenfunction in $B(0,1+\delta)$ such that $\left|\phi_{\delta}\right|_{\infty}=\frac{1}{2}$ i.e. $\phi_{\delta}$ satisfies

$$
\begin{cases}F\left[\phi_{\delta}\right]+h(x) \cdot \nabla \phi_{\delta}\left|\nabla \phi_{\delta}\right|^{\alpha}+\left(V(x)+\bar{\lambda}_{\delta}\right) \phi_{\delta}^{1+\alpha}=0 & \text { in } B(0,1+\delta), \\ \phi_{\delta}=0 & \text { on } \partial B(0,1+\delta) .\end{cases}
$$

For $\epsilon_{1}=\frac{\bar{\lambda}_{\delta}}{2} \min _{B(0,1)} \phi_{\delta}^{1+\alpha}$, we then have

$$
F\left[\phi_{\delta}\right]+h(x) \cdot \nabla \phi_{\delta}\left|\nabla \phi_{\delta}\right|^{\alpha}+\left(V(x)+\bar{\lambda}_{\delta}\right) \phi_{\delta}^{1+\alpha} \leq-2 \epsilon_{1} \quad \text { in } B(0,1) .
$$

Since $u(0)>1$, the function $\chi=u(0) \phi_{\delta}$ satisfies (4.10) as $\phi_{\delta}$. We assume that $\epsilon \leq \epsilon_{1}$, then

$$
F[\chi]+h(x) \cdot \nabla \chi|\nabla \chi|^{\alpha}+\left(V(x)+\bar{\lambda}_{\delta}\right) \chi^{1+\alpha} \leq f(x)-\epsilon_{1} \quad \text { in } B(0,1) .
$$

Let $G$ denote the connected component of the set $\{x \in B(0,1), \mid u(x)>$ $\chi(x)\}$ which contains 0 . Observe that $G$ contains at least one point, say $P_{o}$ on $\partial B(0,1)$. Indeed, if not, $\bar{G}$ would be contained in the interior of $B(0,1)$. Since $u=\chi$ on $\partial G \cap B(0,1)=\partial G$, Theorem 2.3 applied in the set $G$, implies that $u \leq \chi$ in $G$. This is a contradiction since it would imply that $u(0) \leq \frac{u(0)}{2}$. Without loss of generality one may suppose that $P_{o}=(0,1)$.

For $i=1,2,3$, we now introduce $E_{i}$ given by:

$$
\begin{aligned}
& E_{1}=\left\{\left(x_{1}, x_{2}\right), \frac{\left(x_{1}+\frac{5}{2}\right)^{2}}{9}+4\left(x_{2}-\frac{\sqrt{3}}{4}\right)^{2} \leq 1, x_{1} \geq-1\right\}, \\
& E_{2}=\left\{\left(x_{1}, x_{2}\right), \frac{\left(x_{1}-\frac{5}{2}\right)^{2}}{9}+4\left(x_{2}-\frac{\sqrt{3}}{4}\right)^{2} \leq 1, x_{1} \leq 1\right\}, \\
& E_{3}=\left\{\left(x_{1}, x_{2}\right), 4 x_{1}^{2}+\left(\frac{x_{2}-1-\frac{\sqrt{3}}{2}}{2+\frac{\sqrt{3}}{2}}\right)^{2} \leq 1, x_{2} \leq \sqrt{3} / 4\right\} .
\end{aligned}
$$




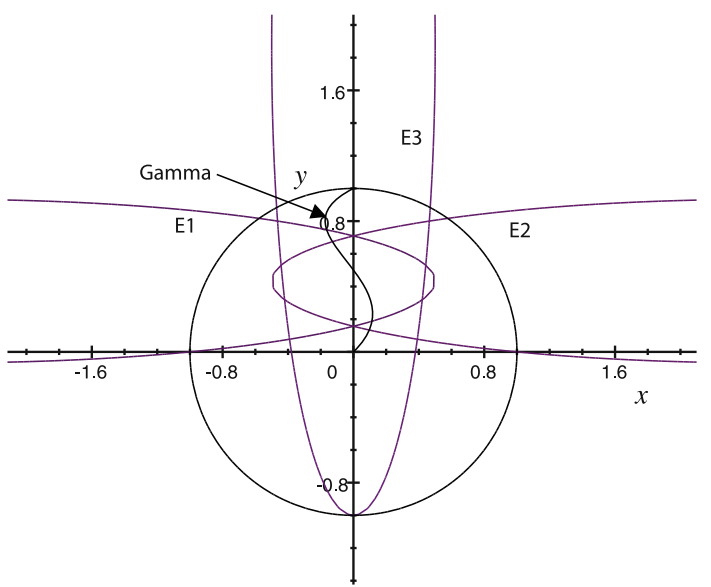

Observe that $B\left(0, \frac{1}{3}\right)$ is contained in the interior of $E_{3}$ and that the boundary $\partial E_{3}$ consists of the elliptic part $\partial E_{3} \cap\left\{\left(x_{1}, x_{2}\right) \mid x_{2}<\frac{\sqrt{3}}{4}\right\}$ and the line segment

$$
L_{3}:=\partial E_{3} \cap\left\{\left(x_{1}, x_{2}\right) \mid x_{2}=\frac{\sqrt{3}}{4}\right\}=\left[-\frac{1}{2}, \frac{1}{2}\right] \times\left\{\frac{\sqrt{3}}{4}\right\} .
$$

Furthermore the straight segment $L_{3}$ is contained in the interior of $E_{1} \cap E_{2}$.

For $i=1,2,3$ let $v_{i}$, be the functions given in Lemma 4.1. We recall that $0 \leq v_{i} \leq 1, v_{i}=0$ on the elliptic boundary of $E_{i}$ and there exists a constant $\varepsilon_{2}>0$ such that $v_{i}$ satisfies

$$
F\left[v_{i}\right]+h(x) \cdot \nabla v_{i}\left|\nabla v_{i}\right|^{\alpha}+V(x) v_{i}^{\alpha+1}>\epsilon_{2} .
$$

Let $\varphi:[0,1] \rightarrow G$ be a simple and regular curve which connects $(0,0)$ and $(0,1)$ i.e. $\varphi(0)=(0,0)$ and $\varphi(1)=(0,1)$. We denote by $\Gamma$ the image of $\varphi$ and define $E=E_{1} \cup E_{2}$. We also introduce

$$
\partial E^{+}=\left\{\left(x_{1}, x_{2}\right) \in \partial E\left|x_{2}>\frac{\sqrt{3}}{4},\right| x_{1} \mid<1\right\}
$$

and

$$
\partial E^{-}=\left\{\left(x_{1}, x_{2}\right) \in \partial E\left|x_{2}<\frac{\sqrt{3}}{4},\right| x_{1} \mid<1\right\} .
$$

Necessarily $\Gamma$ intersects $\partial E^{+}$and $\partial E^{-}$. Let $t^{-}=\sup \left\{t, \varphi(t) \in \partial E^{-}\right\}$and $t^{+}=\inf \left\{t, \varphi(t) \in \partial E^{+}\right\}$. Note that the portion $\varphi(] t^{-}, t^{+}[)$of the curve $\Gamma$ is in the interior of $E$ and that this portion of curve separates $E$ in two parts, the left part $E_{l}$ and the right part $E_{r}$.

Let $D_{1}:=E_{1} \cap E_{r}$ and $D_{2}:=E_{2} \cap E_{l}$, and note that $E_{1} \cap E_{2} \subset D_{1} \cup D_{2}$,

$$
\begin{aligned}
\partial D_{1}= & \left(\varphi(] t^{-}, t^{+}[) \cap D_{1}\right) \\
& \cup\left(\partial E_{1} \cap D_{1}\right) \text { and } \partial D_{2}=\left(\varphi(] t^{-}, t^{+}[) \cap D_{2}\right) \cup\left(\partial E_{2} \cap D_{2}\right) .
\end{aligned}
$$


Setting $\kappa_{1}:=\inf _{B(0,1)} \phi_{\delta}>0$, we have

$$
\begin{aligned}
& u-\kappa_{1} u(0) v_{1}>\kappa_{1} u(0)\left(1-v_{1}\right)>0 \quad \text { on } \varphi(] t^{-}, t^{+}[) \cap D_{1}, \\
& u-\kappa_{1} u(0) v_{1}=u>0 \quad \text { on } \partial E_{1} \cap D_{1} .
\end{aligned}
$$

Analogously

$$
\begin{aligned}
& u-\kappa_{1} u(0) v_{2}>\kappa_{1} u(0)\left(1-v_{2}\right)>0 \quad \text { on } \varphi(] t^{-}, t^{+}[) \cap D_{2}, \\
& u-\kappa_{1} u(0) v_{2}=u>0 \quad \text { on } \partial E_{2} \cap D_{2} .
\end{aligned}
$$

For $i=1,2$ we define $w_{i}=\kappa_{1} u(0) v_{i}$ which satisfies

$$
F\left[w_{i}\right]-|h|_{\infty}\left|\nabla w_{i}\right|^{1+\alpha}-|V|_{\infty} w_{i}^{1+\alpha}>\left(\kappa_{1} u(0)\right)^{1+\alpha} \epsilon_{2} \quad \text { in } E_{i} .
$$

In addition to the first condition on $\epsilon$ we then assume that $\epsilon \leq \kappa_{1}^{1+\alpha} \epsilon_{2}$ so that

$$
F\left[w_{i}\right]-|h|_{\infty}\left|\nabla w_{i}\right|^{1+\alpha}-|V|_{\infty} w_{i}^{1+\alpha}>f(x) \quad \text { in } E_{i}, i=1,2 .
$$

The comparison principle in Theorem 2.3 gives

$$
u(x) \geq \kappa_{1} u(0) \min \left\{v_{1}(x), v_{2}(x)\right\} \quad \text { for all } x \in E_{1} \cap E_{2} .
$$

In particular, setting

$$
\kappa_{2}=\min _{x \in L_{3}}\left(\min \left\{v_{1}(x), v_{2}(x)\right\}\right),
$$

and recalling that $L_{3}$ is contained in the interior of $E_{1} \cap E_{2}$, we have

$$
\min _{x \in L_{3}} u(x) \geq \kappa_{1} \kappa_{2} u(0) .
$$

Choose $w_{3}=\kappa_{1} \kappa_{2} u(0) v_{3}$ so that

$$
F\left[w_{3}\right]-|h|_{\infty}\left|\nabla w_{3}\right|^{1+\alpha}-|V|_{\infty} w_{3}^{1+\alpha}>\left(\kappa_{1} \kappa_{2} u(0)\right)^{1+\alpha} \epsilon_{2} \quad \text { in } E_{3} .
$$

$$
u \geq w_{3} \quad \text { on } L_{3} \quad \text { and } \quad u>0=w_{3} \quad \text { on } \partial E_{3} \cap\left\{x_{2}<\frac{\sqrt{3}}{4}\right\},
$$

i.e. $w_{3} \leq u$ on $\partial E_{3}$. We are in a position to use the comparison principle in Theorem 2.3: assuming that $\epsilon \leq \min \left\{\epsilon_{1},\left(\kappa_{1} \kappa_{2}\right)^{1+\alpha} \epsilon_{2}\right\}$, we get that $u \geq w_{3}$ in $E_{3}$. Since $B\left(0, \frac{1}{3}\right) \subset E_{3}$, we have finally obtained

$$
\min _{B\left(0, \frac{1}{3}\right)} u \geq \kappa_{1} \kappa_{2} u(0) \min _{B\left(0, \frac{1}{3}\right)} v_{3}
$$

Let $K=\left(\kappa_{1} \kappa_{2} \min _{B\left(0, \frac{1}{3}\right)} v_{3}\right)^{-1}$

$$
u(0) \leq K \min _{B\left(0, \frac{1}{3}\right)} u .
$$

In particular, since $K>1$, we have, in both cases $u(0) \leq 1$ and $u(0) \geq 1$,

$$
u(0) \leq K\left(\min _{B\left(0, \frac{1}{3}\right)} u+1\right) .
$$


Now we remove the restriction $|f|_{\infty} \leq \epsilon$. If $|f|_{\infty} \geq \epsilon$, let $v=\left(\frac{\epsilon}{|f|_{\infty}}\right)^{\frac{1}{1+\alpha}} u$, observe that $v$ satisfies

$$
F[v]+h(x) \cdot \nabla v|\nabla v|^{\alpha}+V(x) v^{1+\alpha}=\frac{\epsilon f}{|f|_{\infty}} .
$$

By (4.9), v satisfies:

$$
v(0) \leq K\left(\min _{B\left(0, \frac{1}{3}\right)} v+1\right)
$$

Therefore

$$
u(0) \leq K\left(\min _{B\left(0, \frac{1}{3}\right)} u+\left(\frac{|f|}{\epsilon}\right)^{\frac{1}{1+\alpha}}\right)
$$

Hence

$$
u(0) \leq K \max \left(1, \epsilon^{-\frac{1}{1+\alpha}}\right)\left(\min _{B\left(0, \frac{1}{3}\right)} u+|f|_{\infty}^{\frac{1}{1+\alpha}}\right) .
$$

This ends the proof of the Claim by identifying $K$ and $K \max \left(1, \epsilon^{-\frac{1}{1+\alpha}}\right)$.

Remark that the inequality (4.9) is equivalent to

$$
u(0)+\frac{K}{K-1}|f|_{\infty}^{\frac{1}{1+\alpha}} \leq K\left(u(x)+\frac{K}{K-1}|f|_{\infty}^{\frac{1}{1+\alpha}}\right) .
$$

Fix any point $\bar{x}$ in $B\left(0, \frac{1}{4}\right)$. Then

$$
B\left(\bar{x}, \frac{3}{4}\right) \subset B(0,1), \quad \text { and } \quad 0 \in B\left(\bar{x}, \frac{1}{4}\right) .
$$

Hence, using once again (4.9):

$$
u(\bar{x})+\frac{K}{K-1}|f|_{\infty}^{\frac{1}{1+\alpha}} \leq K\left(u(0)+\frac{K}{K-1}|f|_{\infty}^{\frac{1}{1+\alpha}}\right) .
$$

Using once more $(4.9)$, for all $\bar{y} \in B\left(0, \frac{1}{4}\right)$

$$
u(0)+\frac{K}{K-1}|f|_{\infty}^{\frac{1}{1+\alpha}} \leq K\left(u(\bar{y})+\frac{K}{K-1}|f|_{\infty}^{\frac{1}{1+\alpha}}\right) .
$$

Inserting (4.12) in the inequality above we finally obtain:

$$
\sup _{B\left(0, \frac{1}{4}\right)} u+\frac{K}{K-1}|f|_{\infty}^{\frac{1}{1+\alpha}} \leq K^{2}\left(\inf _{B\left(0, \frac{1}{4}\right)} u+\frac{K}{K-1}|f|_{\infty}^{\frac{1}{1+\alpha}}\right)
$$

and then

$$
\sup _{B\left(0, \frac{1}{4}\right)} u \leq K^{2} \inf _{B\left(0, \frac{1}{4}\right)} u+(K+1) K|f|_{\infty}^{\frac{1}{1+\alpha}} .
$$

Now we consider the case where $\Omega=B(0, R)$ and $u$ is a positive solution of (3.3). Using the homogeneity of the equation $v(x):=u(R x)$ satisfies

$$
\begin{aligned}
& F\left(R x, \nabla v, D^{2} v\right)+R h(R x) \cdot \nabla v|\nabla v|^{\alpha} \\
& \quad+R^{\alpha+2} V(R x) v^{\alpha+1}=R^{2+\alpha} f(R x) \text { in } B(0,1) .
\end{aligned}
$$


By (4.12), with $h$ replaced by $R h(R x)$ and $V(x)$ replaced by $R^{\alpha+2} V(R x), v$ satisfies, for any $\bar{x} \in B\left(0, \frac{1}{3}\right)$ :

$$
v(0)+R^{\frac{\alpha+2}{\alpha+1}} \frac{K}{K-1}|f|_{\infty}^{\frac{1}{1+\alpha}} \leq K\left(v(\bar{x})+R^{\frac{\alpha+2}{\alpha+1}} \frac{K}{K-1}|f|_{\infty}^{\frac{1}{1+\alpha}}\right)
$$

i.e.

$u(0)+R^{\frac{\alpha+2}{\alpha+1}} \frac{K}{K-1}|f|_{\infty}^{\frac{1}{1+\alpha}} \leq K\left(u(\bar{x})+R^{\frac{\alpha+2}{\alpha+1}} \frac{K}{K-1}|f|_{\infty}^{\frac{1}{1+\alpha}}\right) \quad$ for $\bar{x} \in B\left(0, \frac{R}{3}\right)$.

Observe that $K$ depends on $\gamma$ (see (4.8)), but when $R \leq R_{o}$ it can be chosen independently on $R$.

For a general bounded domain $\Omega$ we follow a standard procedure in potential theory. Let $\Omega^{\prime}$ an open set such that $\Omega^{\prime} \subset \subset \Omega$. Let $R=\inf \left\{r, \sup _{x \in \bar{\Omega}^{\prime}} d(x\right.$, $\partial \Omega) \leq r\}$. There exists $k \in \mathbf{N}$, such that for any pair $(\bar{x}, \underline{x})$ of points in $\bar{\Omega}^{\prime}$, and for at most $k$ points $\bar{x}=x_{1}, x_{2}, \ldots, x_{k}=\underline{x}$ the following holds:

$$
x_{i} \in \Omega^{\prime},\left|x_{i}-x_{i+1}\right| \leq \frac{R}{4}, \quad B\left(x_{i}, R\right) \subset \Omega .
$$

Hence applying the previous results, observing that

$$
\bar{\lambda}(\Omega)<\bar{\lambda}\left(B\left(x_{i}, R\right)\right),
$$

we get, for some constant $\beta$ which depends on $K$ and $R$,

$$
\begin{aligned}
u(\bar{x})+\beta|f|_{\infty}^{\frac{1}{1+\alpha}} & \leq K\left(u\left(x_{2}\right)+\beta|f|_{\infty}^{\frac{1}{1+\alpha}}\right) \\
& \leq K^{2}\left(u\left(x_{3}\right)+\beta|f|_{\infty}^{\frac{1}{1+\alpha}}\right) \leq K^{k}\left(u(\underline{x})+\beta|f|_{\infty}^{\frac{1}{1+\alpha}}\right) .
\end{aligned}
$$

Since this inequality holds for any $\bar{x}, \underline{x}$ in $\Omega^{\prime}$, this ends the proof of Theorem 3.1.

Proof of Corollary 3.2. Suppose that $u$ is a solution in $\Omega$ which contains $B\left(0, R_{o}\right)$. Let $v$ be defined as $v(x)=: u(R x)$. Then $v$ satisfies in $B\left(0, \frac{R_{o}}{R}\right)$

$F\left(R x, \nabla v(x), D^{2} v(x)\right)+R h(R x) \cdot \nabla v|\nabla v|^{\alpha}+R^{2+\alpha} V(R x) v^{1+\alpha}=R^{2+\alpha} f(R x)$.

Applying (4.9) for $v$, we get (3.5) for $u$.

We now prove that $u$ is Hölder's continuous.

Let $R_{o}>0$ such that $B\left(x_{o}, 4 R_{o}\right) \subset \Omega^{\prime} \subset \subset \Omega$. We define for any $R<R_{o}$

$$
M_{i}=\max _{B\left(x_{o}, i R\right)} u, \quad m_{i}=\min _{B\left(x_{o}, i R\right)} u
$$

for $i=1$ and $i=4$. Then $u-m_{i}$ is a solution of

$$
F\left[u-m_{i}\right]+h(x) \nabla\left(u-m_{i}\right)\left|\nabla\left(u-m_{i}\right)\right|^{\alpha}=-V(x) u^{1+\alpha}+f(x)
$$

in $B\left(x_{o}, i R\right)$, hence $u$ satisfies

$$
\sup _{B\left(x_{o}, R\right)}\left(u(x)-m_{4}\right) \leq K \inf _{B\left(x_{o}, R\right)}\left(u(x)-m_{4}\right)+K R^{\frac{2+\alpha}{\alpha+1}}\left(M_{4}|V|_{\infty}^{\frac{1}{1+\alpha}}+|f|_{\infty}^{\frac{1}{1+\alpha}}\right) .
$$


In the same way, using the operator $G(x, p, M)=-F(x, p,-M)$, and the function $M_{i}-u$, we get in $B(0, i R)$,

$$
G\left(x, \nabla u, D^{2}\left(M_{i}-u\right)\right)+h(x) \cdot\left|\nabla\left(M_{i}-u\right)\right|^{\alpha} \nabla\left(M_{i}-u\right)=V(x) u^{1+\alpha}-f(x) .
$$

For $K$ as above, we have obtained that

$$
\sup _{B\left(x_{o}, R\right)}\left(M_{4}-u(x)\right) \leq K \inf _{B\left(x_{o}, R\right)}\left(M_{4}-u(x)\right)+K R^{\frac{2+\alpha}{\alpha+1}}\left(M_{4}|V|_{\infty}^{\frac{1}{1+\alpha}}+|f|_{\infty}^{\frac{1}{1+\alpha}}\right) .
$$

Summing this inequality with (4.13), for some constant $K^{\prime}$ independent of $R \leq R_{o}$, we obtain

$$
M_{1}-m_{1} \leq \frac{K-1}{K+1}\left(M_{4}-m_{4}\right)+K^{\prime} R^{\frac{2+\alpha}{\alpha+1}} .
$$

The rest of the proof is classical, just apply Lemma 8.23 in [19].

Proof of Corollary 3.3. Let $c_{0}=\inf _{\mathbb{R}^{2}} u$ and let $w=u-c_{0}$. Clearly $w$ satisfies in $\mathbb{R}^{2}$ :

$$
F[w]=0, \quad w \geq 0, \quad \inf w=0 .
$$

Suppose by contradiction that $w>0$ somewhere, then applying the strong maximum principle one gets that $w>0$ in the whole of $\mathbb{R}^{2}$.

By definition of the infimum, for any $\varepsilon>0$ there exists $\underline{x} \in \mathbb{R}^{2}$ such that $w(\underline{x}) \leq \varepsilon$. Now for any $x \in \mathbb{R}^{2}$ consider the ball $B(\underline{x}, 4|x-\underline{x}|)$, by Harnack's inequality, we get that

$$
w(x) \leq K w(\underline{x}) \leq K \varepsilon .
$$

Observe that $K$ does not depend on the distance $|x-\underline{x}|$ because $h=V \equiv 0$, hence it does not depend on the choice of $x$. Since this holds for any $\varepsilon$ we get $w \equiv 0$.

\section{Acknowledgments}

The authors are very grateful to the anonymous referee for the very interesting comments and the considerable improvements she/he brought to the proof of Harnack's inequality.

\section{References}

[1] Berestycki, H., Nirenberg, L., Varadhan, S.R.S.: The principal eigenvalue and maximum principle for second-order elliptic operators in general domains. Comm. Pure Appl. Math. 47(1), 47-92 (1994)

[2] Berestycki, H., Rossi, L.: On the principal eigenvalue of elliptic operators in $\mathbb{R}^{N}$ and applications. J. Eur. Math. Soc. 8(2), 195-215 (2006)

[3] Birindelli, I., Demengel, F.: Comparison principle and Liouville type results for singular fully nonlinear operators. Ann. Fac. Sci. Toulouse Math. (6) 13, 261287 (2004) 
[4] Birindelli, I., Demengel, F.: Eigenvalue and Maximum principle for fully nonlinear singular operators. Adv. Partial Differ. Equ. 11(1), 91-119 (2006)

[5] Birindelli, I., Demengel, F.: Eigenvalue, maximum principle and regularity for fully non linear homogeneous operators. Comm. Pure Appl. Anal. 6, 335$366(2007)$

[6] Birindelli, I., Demengel, F.: The Dirichlet problem for singular fully nonlinear operators. Discrete and Cont. Dynamical Syst. (2007), Special vol. 110-121

[7] Busca, J., Esteban, M.J., Quaas, A.: Nonlinear eigenvalues and bifurcation problems for Pucci's operator. Ann. Inst. H. Poincaré, Anal. Linéaire 22, $187-$ $206(2005)$

[8] Busca, J., Sirakov, B.: Harnack type estimates for nonlinear elliptic equations systems and applications. Ann. Inst. H. Poincaré, Anal. Linéaire 21, 543$590(2004)$

[9] Cabré, X.: On the Alexandroff-Bakelman-Pucci estimate and the reversed Hölder inequality for solutions of elliptic and parabolic equations. Commun. Pure Appl. Math. 48(5), 539-570 (1995)

[10] Capuzzo-Dolcetta, I., Leoni, F., Vitolo, A.: The Alexandrov-BakelmanPucci weak maximum principle for fully nonlinear equations in unbounded domains. Commun. Partial Differ. Equ. 30(10-12), 1863-1881 (2005)

[11] Capuzzo Dolcetta, I., Vitolo, A.: A qualitative Phragmn-Lindelöf theorem for fully nonlinear elliptic equations. J. Differ. Equ. 243(2), 578-592 (2007)

[12] Caffarelli, L., Cabré, X.: Fully-nonlinear equations Colloquium Publications 43. American Mathematical Society, Providence (1995)

[13] Cutrì, A., Leoni, F.: On the Liouville property for fully nonlinear equations. Ann. Inst. H. Poincaré Anal. Non Linéaire 17, 219-245 (2000)

[14] Davila, G., Felmer, P., Quaas, A.: Harnack Inequality For Singular Fully Nonlinear Operators and some existence results, Preprint

[15] Davila, G., Felmer, P., Quaas, A.: Alexandroff-Bakelman-Pucci estimate for singular or degenerate fully nonlinear elliptic equations. C. R. Math. Acad. Sci. Paris 347 (2009)

[16] Delarue, F.: Krylov and Safonov estimates for degenerate quasilinear elliptic PDEs, preprint

[17] Imbert, C.: Alexandroff-Bakelman-Pucci estimate and Harnack inequality for degenerate fully nonlinear elliptic equations, preprint

[18] Ishii, H., Yoshimura, Y.: Demi-eigenvalues for uniformly elliptic Isaacs operators, preprint

[19] Gilbarg, D., Trudinger, N.S.: Elliptic Partial Differential equations of second order, 2nd edn. Springer, Berlin (1983) 
[20] Krylov, N.V., Safonov, M.V.: An estimate for the probability of a diffusion process hitting a set of positive measure. (Russian) Dokl. Akad. Nauk SSSR 245(1), 18-20 (1979)

[21] Krylov, N.V., Safonov, M.V.: A certain property of solutions of parabolic equations with measurable coefficients. Izv. Akad. Nauk SSSR Ser. Mat. 44(1), 161$175(1980)$

[22] Lions, P.-L.: Bifurcation and optimal stochastic control. Nonlinear Anal. 7(2), 177-207 (1983)

[23] Quaas, A., Sirakov, B.: Principal eigenvalues and the Dirichlet problem for fully nonlinear elliptic operators. Adv. Math. 218(1), 105-135 (2008)

[24] Quaas, A., Sirakov, B.: On the principal eigenvalues and the Dirichlet problem for fully nonlinear operators. C. R. Math. Acad. Sci. Paris 342(2), 115-118 (2006)

[25] Serrin, J.: On the Harnack inequality for linear elliptic equations. J. Anal. Math. 4, 292-308 (1955/56)

[26] Serrin, J.: Local behavior of solutions of quasi-linear equations. Acta Math. 111(1), 247-302 (1964)

[27] Trudinger, N.S.: On Harnack type inequalities and their application to quasilinear elliptic equations. Comm. Pure Appl. Math. 20, 721-747 (1967)

I. Birindelli

Universita di Roma La Sapienza

Rome, Italy

e-mail: isabeau@mat.uniroma1.it

F. Demengel

Université de Cergy-Pontoise

Cergy-Pontoise, France

Received: 13 May 2009.

Accepted: 10 May 2010. 